\title{
CORRIGENDUM
}

\section{Origami structures with a critical transition to bistability arising from hidden degrees} of freedom

Jesse L. Silverberg, Jun-Hee Na, Arthur A. Evans, Bin Liu, Thomas C. Hull, Christian D. Santangelo, Robert J. Lang, Ryan C. Hayward and Itai Cohen

Nature Materials 14, 389-393 (2015); published online 9 March 2015; corrected after print 31 March 2015.

In the version of this Letter originally published, the authors Jesse L. Silverberg and Jun-Hee Na should have been denoted as having contributed equally to this work. This has now been corrected in the online versions of the Letter. 